\title{
Ensuring the Continuity of Care of Cardiorespiratory Diseases at Home: Monitoring Equipment and Medical Data Exchange over Semantically Annotated Web Services
}

\section{B. Spyropoulos', A. Tzavaras', M. Botsivaly ${ }^{1}$, K. Koutsourakis ${ }^{1}$}

${ }^{1}$ Medical Instrumentation Technology Department, Faculty of Engineering, Technological Educational Institution (TEI) of Athens, Athens, Greece

\section{Summary \\ Objectives}

A significant portion of care related to cardiorespiratory diseases is provided at home, usually but not exclusively, after the discharge of a patient from hospital. It is the purpose of the present study to present the technical means which we have developed, in order to support the adaptation of the continuity of care of cardiorespiratory diseases at home.

\section{Methods}

We have developed an integrated system that includes: first, a prototype laptopbased portable monitoring system that comprises low-cost commercially available components, which enable the periodical or continuous monitoring of vital signs at home; second, software supporting medical decision-making related to tachycardia and ventricular fibrillation, as well as fuzzy-rules-based software supporting home-ventilation optimization; third, a typical continuity of care record (CCR) adapted to support also the creation of a homecare plan; and finally, a prototype ontology, based upon the HL7 clinical document architecture (CDA), serving as basis for the development of semantically annotated web services that allow for the exchange and retrieval of homecare information.

\section{Results}

The flexible design and the adaptable data-exchange mechanism of the developed system result in a useful and standard-compliant tool, for cardiorespiratory disease-related homecare.

\section{Conclusions}

The ongoing laboratory testing of the system shows that it is able to contribute to an effective and low-cost package solution, supporting patient supervision and treatment. Furthermore, semantic web technologies prove to be the perfect solution for both the conceptualization of a continuity of care data exchange procedure and for the integration of the structured medical data.

\section{Keywords \\ continuity of care, Homecare, cardio- respiratory diseases, semantically annotated web-services \\ DOI \\ 10.3414/ME9305 \\ The full English version can be found in /Methods of Information in Medicine 2010 49 2: $156-160 /$ or http://www.schattauer.de/de/magazine/ue bersicht/zeitschriften-a- z/methods/issue/special/manuscript/1261 6/show.html.}

